\title{
Determinación de propiedades elásticas de piezas poliméricas construidas por impresión 3D, sometidas a flexión
}

\section{Determination of elastic properties of polymeric pieces constructed by 3D printing, subjected to bending}

\author{
Darío Stechina ${ }^{1}$, Sandra Marina Mendoza ${ }^{2}$, \\ Héctor Daniel Martín ${ }^{1}$, Claudio Norberto Maggi ${ }^{1}$ \\ Marcelo Tulio Piovan ${ }^{3}$
}

\footnotetext{
${ }^{1}$ Universidad Tecnológica Nacional, Facultad Regional Reconquista, Calle 40 Nº1000, 3560 Reconquista, Santa Fe, Argentina. e-mail: dario.stechina@gmail.com

${ }^{2}$ Universidad Tecnológica Nacional, CONICET, Facultad Regional Reconquista, Calle 40, n. 1000, 3560 Reconquista, Santa Fe, Argentina.

${ }^{3}$ Universidad Tecnológica Nacional, CONICET, Facultad Regional Bahía Blanca, 11 de abril 461, 8000 Bahía Blanca, Buenos Aires, Argentina.

e-mail: smendoza@frrq.utn.edu.ar,hmartin@frrq.utn.edu.ar,ncmaggi@arnet.com.ar,mpiovan@ frbb.utn.edu.ar
}

\section{RESUMEN}

El procedimiento de Modelación por Deposición de Filamento (MDF) polimérico es una de las variantes de mayor repercusión en el conjunto de tecnologías de construcción aditiva, usualmente llamadas impresión 3D. La irrupción de máquinas de bajo costo y la continua mejora de la calidad y resistencia de los polímeros empleados, exige estimar la vida útil y performance de las piezas impresas.

Por ello, este trabajo analiza el desempeño mecánico ante esfuerzos de flexión de polímeros que típicamente se emplean en la construcción aditiva. En particular se evalúa, a través de experimentos diseñados "ad-hoc" el módulo de elasticidad a flexión en probetas de Poli Ácido Láctico (PLA), Nylon y Poli-estireno de Alto Impacto (HIPS), en función de diversos factores de variabilidad, entre los que se cuentan la dirección de trazado en cada capa (ángulo de deposición), la orientación de impresión de la pieza, la presencia de aditivos colorantes en los polímeros y la velocidad de impresión.

Se encontró que todos los factores de variabilidad estudiados, modifican significativamente el módulo de elasticidad a la flexión. Analizando la orientación de los filamentos en los tres materiales, se determinó que la técnica MDF produce piezas anisótropas. El mayor valor del módulo de elasticidad se logra cuando la dirección de todos los filamentos coincide con la dirección de los esfuerzos principales. A su vez la coloración de los materiales en crudo tiene influencia significativa como también la dirección en la que se elabora la impresión. También se comprobó que el módulo de elasticidad disminuye al aumentar la velocidad de impresión.

Palabras clave: Impresión 3D; Flexión; PLA; HIPS; Nylon.

\section{ABSTRACT}

The method of Fused Deposition Modelling (MDF) for polymers processing - usually called 3D printing - is one of the additive construction technologies with greatest impact in recent years. The irruption of low cost machines and the continuous improvement in quality and resistance of employed polymers, demand to estimate the life time and performance of printed parts.

Hence, this work analyses the mechanical performance under bending efforts of polymers, typically used in additive construction. In particular, the bending elastic modulus is evaluated - through experiments designed "ad-hoc" - in Poly Lactic Acid (PLA), Nylon, and High Impact Polystyrene (HIPS) probes, as a function of tracing direction, orientation of printed parts, presence of dye additives in polymers, and printing speed. 
We found that all the studied variability factors significantly modify the bending elastic modulus. Analysis of filaments orientation in the three materials reveals that the MDF technique produces anisotropic pieces. The highest elastic modulus is achieved when all filament directions match the direction of main stresses. Besides, the dye additives have significant influence as well as the orientation of the printed parts. Besides, we verified that the elastic modulus decreases with increasing printing speed.

Keywords: 3D Printing; Bending; PLA; HIPS; Nylon.

\section{INTRODUCCIÓN}

La tecnología de construcción aditiva, coloquialmente llamada impresión 3D, se ha convertido en una de las principales herramientas para fabricar todo tipo de componentes y bienes. Su implementación abarca un espectro que va desde la simple escala doméstica hasta la sofisticación industrial [1]. De entre la gran variedad de procedimientos de construcción aditiva, el denominado Modelado por Deposición de Filamento (MDF) es el más difundido. Entre sus principales ventajas se destaca mantener la versatilidad operativa, el bajo costo de las máquinas y de los insumos empleados - generalmente polímeros termoplásticos -. El MDF fue concebido e implementado industrialmente en la década de 1980. En sus inicios, se utilizaba para facilitar la visualización rápida de prototipos industriales en materiales plásticos, como paso previo al diseño y construcción definitiva en materiales metálicos, los que seguían procedimientos convencionales de mecanizado. Por esa razón, no se necesitaba evaluar la respuesta mecánica ni caracterizar aspectos constitutivos/elásticos de tales prototipos plásticos, puesto que se descartaban. Durante los últimos años - bajo las urgencias de reducción de costos y optimización de tiempos producción, la irrupción de máquinas muy económicas y la mejora de la resistencia y calidad de los insumos poliméricos - el MDF se vislumbra como una alternativa sustancialmente útil para fabricar componentes funcionales de compleja geometría. Esto incrementó el interés de la comunidad tecnológica y científica, incentivando la caracterización de propiedades elásticas de componentes impresos y la construcción de modelos constitutivos para diversos tipos de plásticos [2]-[10].

La construcción aditiva por MDF se puede configurar para lograr piezas que, aun teniendo la misma forma volumétrica, presenten propiedades y respuestas mecánicas disímiles. Es así que la disposición de los filamentos en una capa puede asemejarse a una lámina de material compuesto (con una orientación resistente preferencial), o bien poseer una configuración en panal de abeja, o tener refuerzos estructurales internos, o la configuración que el usuario desee. Se puede dotar así a la estructura de una respuesta racionalmente prescrita. Sin embargo, para ello es necesario caracterizar las propiedades elásticas requeridas para construir modelos de cálculo y estimar la respuesta estructural ante un diseño de pieza en particular [11]-[13].

Durante los últimos 15 años se han efectuado estudios de caracterización de propiedades elásticas y mecánicas [2]-[16], en general sobre piezas construidas mediante técnicas de impresión 3D por deposición de filamento plástico. En algunos casos se han propuesto modelos matemáticos sencillos para representar la resistencia y la rigidez de los filamentos en crudo, filamentos extruidos y fundamentalmente de las piezas impresas. En tales estudios se han empleado criterios de ensayo que abarcan un espectro muy amplio, donde se utilizaron metodologías estrictamente acordes a normas internacionales (e.g. ISO 178 y D790 para flexión), variantes permitidas por dichas normas y también otros criterios experimentales elaborados "ad hoc". Estos dos últimos criterios son necesarios en virtud de poder salvar algunas contingencias vinculadas a la clase de instrumentos necesarios, a las dimensiones de las probetas y a que las normas antes mencionadas fueron concebidas para piezas plásticas construidas por inyección. En este marco cabe recalcar que las organizaciones de estandarización de materiales (ISO, ASTM, etc.) aún no han elaborado, con suficiente consenso, normas apropiadas para ensayos de piezas impresas. Los institutos de normativas nacionales como DIN y NIST, suelen ofrecer recomendaciones sobre la validez de uso de las normas de ensayo para plásticos inyectados en el contexto de piezas impresas [17].

Casi todos los estudios mencionados más arriba se han centrado en la caracterización de Acrilonitrilo Butadieno Estireno (ABS) y de Poli Ácido Láctico (PLA), que son dos de los polímeros más empleados en MDF desde comienzos de 2000. En otros artículos [8]-[10], [14]-[16] se efectuaron estudios de caracterización de propiedades elásticas comparando y evaluando piezas construidas bajo diversos tipos de máquinas (profesionales de marca registrada, diseños propios y de bajo costo del proyecto RepRap), metodologías experimentales, diversos tipos de características constructivas y/o parámetros operativos.

La influencia de la disposición angular de las capas y el número de capas en el perímetro externo han sido dos parámetros de impresión que se han estudiado desde hace al menos 20 años. En primera instancia, se ha intentado asociar resultados experimentales con formas de modelación semejantes a los materiales compuestos de resinas reforzados por fibras [3]-[5]. Más recientemente se han hecho otros estudios incorporando 
correlaciones entre los diversos parámetros de impresión [6]-[10], [14]-[16]. En ellos se ha observado que la mayor resistencia a tracción se obtiene con los filamentos orientados en la dirección de la solicitación. El espesor de capa es uno de los parámetros más influyentes, y a pesar de ser muy estudiado se revela controvertido [7]-[12]. En efecto, en algunos estudios como el de VAEZI y CHUA [7] y el de TYMRAK et al. [9] se ha afirmado que la disminución del espesor conduce a un incremento de la resistencia a la tracción; mientras que en los estudios de SOOD et al. [16] y de CHACON et al. [15] se ha observado una variabilidad vinculada a otros parámetros de impresión. En el estudio de CHACON et al. [15] se ha empleado el método de análisis de varianza y métodos de regresión, entre otros, para establecer una caracterización paramétrica de las propiedades mecánicas del poli-ácido láctico y determinar configuraciones óptimas de impresión en términos de resistencia y ahorro de tiempo. Las publicaciones internacionales sobre este tema en particular han ido creciendo de manera exponencial en los últimos 4 años. Sin embargo, se desea destacar que varios tópicos no fueron cubiertos en su totalidad y consecuentemente merecen ser analizados. En particular, no se han observado estudios sobre la caracterización de propiedades elásticas de piezas impresas en Nylon y HIPS, entre otros. Parámetros operativos como la velocidad de impresión y su relación con la calidad dimensional y estructural no han sido tratados con suficiente amplitud y detalle, a pesar de haber varios aportes [14]-[16].

En este artículo se examina y analiza la influencia de un conjunto de parámetros de impresión sobre las propiedades elásticas y mecánicas de probetas construidas en los siguientes materiales: PLA, HIPS y Nylon. Las mismas fueron manufacturadas en una impresora 3D de bajo costo, empleando la técnica MDF. Se evalúa la influencia de factores de variación tales como la dirección de trazado en cada capa, la orientación de impresión de la pieza, los pigmentos usados para coloración de los polímeros y la velocidad de impresión. Se efectúan ensayos de flexión de 3 puntos para determinar las características mecánicas de elasticidad.

\section{MATERIALES Y MÉTODOS}

En este trabajo se analizan las características elásticas y mecánicas de especímenes impresos de PLA, Nylon y HIPS. Se utilizaron materiales de la marca registrada High-QS con filamento de $1.75 \mathrm{~mm}$ de diámetro. Los especímenes experimentales se construyeron con una impresora de mesa tipo RepRap variante Prusa-Mendel M90, marca Replikat, modelo M5 [18]. Esta impresora puede ser controlada con diversos programas de código abierto con configuración particular para la misma. A su vez para la generación de los archivos G-Code se pueden emplear varios programas shareware tales como KissSlicer, Slic3r, o CURA, entre otros. La impresora permite el intercambio de boquillas de diversos tamaños y calidades. Aunque para este estudio se empleó solamente la boquilla de $0.4 \mathrm{~mm}$ de diámetro.

Tal como se ha mencionado en el apartado Introducción, todavía no hay normas y metodologías específicas para efectuar ensayos de flexión en partes construidas empleando la técnica MDF. Esto ha generado y aun genera controversias entre autores con respecto a la metodología de ensayo, tipo y forma de probetas empleadas. Sin embargo, se ha observado un consenso en seguir, a falta de algo concreto y específico, las normas (ASTM, ISO, DIN, etc.) disponibles, correspondientes a ensayos de plásticos inyectados y/o las variantes permitidas dentro de las mismas. Se emplea aquí el criterio de la norma ISO 178 [19] para los ensayos de flexión, en consonancia con las hipótesis de trabajo utilizadas en otras publicaciones [6]-[10]. La forma y dimensiones de las probetas se esquematiza en la Figura 1a. El modelo CAD de los especímenes fue realizado en el programa de CAD sólido 123D Design (Freeware de Autodesk) para ser exportado en formato STL y procesado en el programa de rebanado KissSlicer para preparación del G-Code.

La técnica MDF permite construir probetas de una misma geometría y volumen final, siguiendo caminos de impresión diferentes a medida que se deposita cada capa de material. Por ejemplo, la boquilla de impresión puede seguir caminos con diversas orientaciones. En este trabajo se analiza este factor constructivo y para ello se modelaron probetas alternando la orientación de los filamentos en cada capa de material impreso, es decir, depositando filamentos con ángulos relativos $(\beta)$ diferentes entre capas sucesivas (ver Figura 1b). Por ejemplo, si $\beta 1 / \beta 2$ es $0^{\circ} / 0^{\circ}$, significa que todos los filamentos se depositaron siguiendo caminos paralelos a la cara más larga de la probeta en todas las capas de material. La relación $\beta 1 / \beta 2=45^{\circ} / 45^{\circ}$ indica que los filamentos de dos capas sucesivas son perpendiculares entre sí, pero están desfasados del eje longitudinal de la probeta en $45^{\circ}$.

Otro factor de variación es la base de impresión, ya que es posible realizar una probeta para ensayos de flexión - que es un paralelepípedo - (Figura 1a) tomando como base de impresión la superficie de mayor área (ancho, A) o la de menor área (espesor, E), arribando a la misma geometría final (Figura 1c).

En este trabajo se evalúan distintos factores de variación para cada material, que son: la dirección de trazado entre capas, la orientación de la pieza impresa con respecto a su base de impresión, la velocidad de 
impresión y la presencia de aditivos colorantes de la matriz polimérica. Los parámetros constructivos a evaluar utilizados en la impresión de las probetas de PLA, HIPS y Nylon se resumen en las Tablas 1 a 3, respectivamente. La selección de los colores se ha hecho en base a lo disponible en mercado durante el período de ensayos. Sobre este particular, cabe acotar que los autores tan solo pueden guiarse por el color y/o tono del polímero en evaluación, en tanto que los pigmentos que se usan son potestad del registro de marcas, en consecuencia no disponibles. Por otro lado, en la Tabla 4 se indican las condiciones de impresión fijas para cada uno de los materiales, tales como temperaturas de pico y de plataforma, que son las estándares sugeridas por el fabricante. Se indican, a su vez, los detalles que hacen a la calidad de impresión como por ejemplo protocolo de adhesión a la plataforma y sustancia empleada para adherir, además de otros parámetros operativos/constructivos fijos para todo el conjunto.

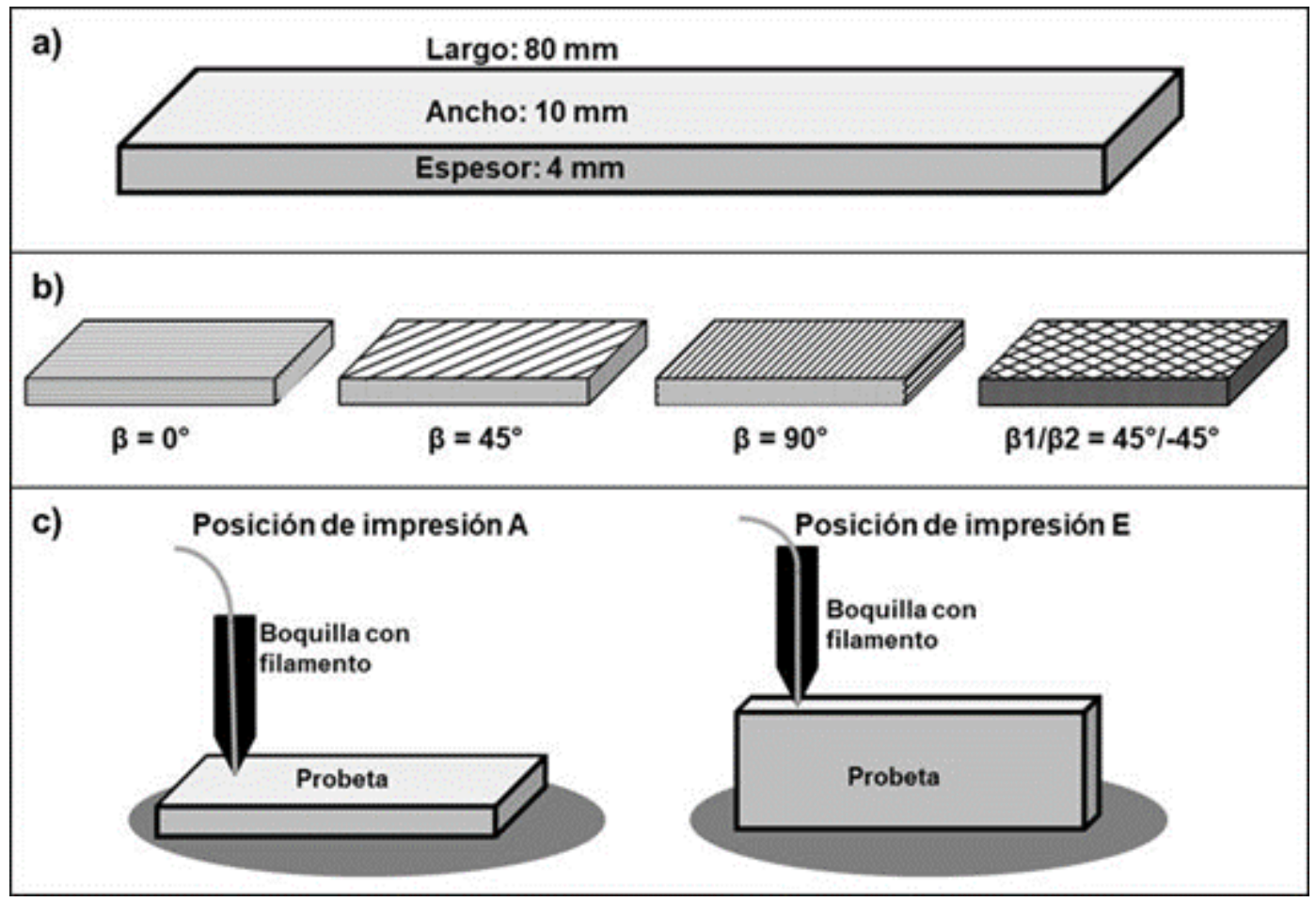

Figura 1: a) Dimensiones de una probeta para ensayos de flexión, según norma ISO 178. b) Representación esquemática de la orientación de deposición de los filamentos con respecto a la geometría de la probeta. $\beta 1 / \beta 2$ indica que la orientación fue alternada en ángulos $\beta 1$ y $\beta 2$ entre capas consecutivas de material depositado. c) Representación esquemática de la posición de la boquilla de impresión con respecto a la superficie de impresión de la probeta.

Tabla 1: Parámetros constructivos que se utilizaron en PLA para imprimir las probetas.

\begin{tabular}{l|l}
\hline PARÁMETROS DE IMPRESIÓN & VALORES UTILIZADOS \\
\hline Velocidad de impresión [mm/s] & $24 ; 32 ; 40$ \\
\hline $\begin{array}{l}\text { Ángulos de orientación del filamento entre dos } \\
\text { capas del tramado de impresión }\left[^{\circ}\right]\end{array}$ & $0 / 0 ; 45 /-45 ; 0 / 90$ \\
\hline Porcentaje de llenado [\%] & 100 \\
\hline Base de impresión & Superficie de mayor (A) o menor área (E). \\
\hline Color & Sin pigmento; gris; azul; celeste; rojo. \\
\hline
\end{tabular}

Tabla 2: Parámetros constructivos que se utilizaron en HIPS para imprimir las probetas.

\begin{tabular}{l|l}
\hline PARÁMETROS DE IMPRESIÓN & VALORES UTILIZADOS \\
\hline Velocidad de impresión [mm/s] & $24 ; 32 ; 40$ \\
\hline Ángulos de orientación del filamento entre dos & $0 / 0 ; 45 /-45 ; 0 / 90$ \\
\hline
\end{tabular}




\begin{tabular}{l|l}
\hline capas del tramado de impresión $\left[^{\circ}\right]$ & \\
\hline Porcentaje de llenado [\%] & 100 \\
\hline Base de impresión & Superficie de mayor (A) o menor área (E). \\
\hline Color & ocre; azul; verde \\
\hline
\end{tabular}

Tabla 3: Parámetros constructivos que se utilizaron en Nylon para imprimir las probetas.

\begin{tabular}{l|l}
\hline PARÁMETROS DE IMPRESIÓN & VALORES UTILIZADOS \\
\hline Velocidad de impresión [mm/s] & $24 ; 32 ; 40$ \\
\hline $\begin{array}{l}\text { Ángulos de orientación del filamento entre dos } \\
\text { capas del tramado de impresión }\left[^{\circ}\right]\end{array}$ & $0 / 0 ; 45 /-45 ; 0 / 90$ \\
\hline Porcentaje de llenado [\%] & 100 \\
\hline Base de impresión & Superficie de mayor (A) o menor área (E). \\
\hline Color & Sin pigmento; negro. \\
\hline
\end{tabular}

Algunos aspectos se desean remarcar con relación a las condiciones de impresión. La disminución en la velocidad de impresión de la primera capa es un procedimiento estándar muy útil para auxiliar a la fijación de la pieza a la plataforma y en consecuencia coadyuvar a la buena calidad de terminación. El procedimiento de fijación seleccionado ha sido el tipo "Brim" o "faldón" con $\mathrm{xN}$ vueltas o perímetros $(\mathrm{N}=4$ o 10 según se requiera). El Nylon es uno de los materiales más difíciles de imprimir debido a su tendencia al alabeo, en este caso se ha empleado PVA (cola vinílica) en barra para mejorar la fijación y evitar el alabeo, además el rollo fue expuesto a un proceso de precalentamiento a $105^{\circ} \mathrm{C}$ en horno durante una hora y mantenido en cámara desecante para evitar presencia espuria de humedad. Además, la impresora se encuentra en un ambiente donde se mantiene condiciones de temperatura y humedad estables tales como las que se observan en la Tabla 4.

Tabla 4: Condiciones experimentales para la impresión de cada uno de los materiales involucrados.

\begin{tabular}{l|l|l|l}
\hline CONDICION & PLA & HIPS & NYLON \\
\hline Temperatura de plataforma $\left[{ }^{\circ} \mathrm{C}\right]$ & $60 \pm 3$ & $80 \pm 5$ & $110 \pm 3$ \\
\hline Temperatura de boquilla $\left[{ }^{\circ} \mathrm{C}\right]$ & $200 \pm 5$ & $235 \pm 5$ & $250 \pm 5$ \\
\hline Protocolo de adhesión a plataforma & Brim $(\mathrm{x} 4)$ & Brim $(\mathrm{x} 4)$ & Brim $(\mathrm{x} 10)$ \\
\hline Sustancia de adhesión a plataforma & Fijador/spray & Fijador/spray & PVA/barra \\
\hline Superposición de filamentos $[\%]$ & 10 & 10 & 10 \\
\hline Reducción Velocidad primera capa $[\%]$ & 80 & 80 & 85 \\
\hline Temperatura ambiente de impresión $\left[{ }^{\circ} \mathrm{C}\right]$ & $22 \pm 4$ & $25 \pm 4$ & $25 \pm 4$ \\
\hline Humedad ambiente de impresión $[\%]$ & $25 \pm 5$ & $25 \pm 5$ & $25 \pm 5$ \\
\hline Espesor de capa $[\mathrm{mm}]$ & 0.2 & 0.2 & 0.2 \\
\hline Cáscara lateral (número de perímetros) & 2 & 2 & 2 \\
\hline
\end{tabular}

Los ensayos de flexión de tres puntos se llevaron a cabo con una máquina universal de ensayos de pequeñas dimensiones, marca PASCO, modelo ME8244. La adquisición de datos se realizó mediante el software PASCO Capstone y las propiedades mecánicas se determinaron a partir de las gráficas de esfuerzo versus deformación. Los valores aquí informados corresponden al promedio de al menos 6 probetas de equivalentes características para cada caso. Además, siempre que se contó con más de 12 probetas equivalentes, los datos fueron evaluados estadísticamente mediante Análisis de la Varianza (ANOVA), con un nivel de significancia de $95 \%$, es decir, un valor $\mathrm{p}=0.05$. Cuando el test ANOVA arrojó variaciones entre los resultados, se utilizaron el test de Tukey y los análisis gráficos en caso de querer individualizar los grupos de probetas diferentes.

\section{RESULTADOS}

La Figura 2(a) muestra los resultados obtenidos a partir de probetas de PLA donde se varía el ángulo de deposición de filamentos, alternando el tramado entre dos capas sucesivas en ángulos $\beta 1 / \beta 2$. Las barras del grá- 
fico corresponden a valores promedio de cada conjunto de mediciones. Se representa en cada una de ellas el error estándar, que permite visualizar la baja dispersión de los valores experimentales. Todos los demás parámetros de impresión de PLA, sin pigmentos en este caso, se mantuvieron constantes y se realizaron con un espesor de capa de $0,2 \mathrm{~mm}, 100 \%$ de llenado, velocidad de $32 \mathrm{~mm} / \mathrm{s}$ y base de impresión en el sentido del ancho (es decir, la superficie de mayor área, A).

Los datos de la Figura 2(a) fueron comparados mediante ANOVA, que confirma diferencias significativas entre los módulos de elasticidad debido a la variación del ángulo de deposición $(\mathrm{p}<0,05)$. Se puede observar que la diferencia estadística se debe al máximo valor, obtenido para el caso $0^{\circ} / 0^{\circ}$, el cual disminuye hasta un $12 \%$ en los casos $45^{\circ}-45$ y $0^{\circ} / 90^{\circ}$. Los resultados sugieren que un desfasaje de los filamentos con respecto a la dirección longitudinal de la probeta es la causa de la disminución del módulo de elasticidad. En otras palabras, la máxima resistencia se logra cuando la dirección de todos los filamentos coincide con la dirección de las máximas tensiones. Estos resultados evidencian que la construcción aditiva mediante MDF resulta en piezas anisótropas con respecto al módulo de elasticidad a la flexión. El hecho es de esperar ya que, durante el proceso MDF, el material se va solidificando a medida que se deposita. La adhesión entre las superficies de dos filamentos de PLA adyacentes no es la misma que entre las moléculas del interior de cada filamento, resultando en uniones más débiles y regiones porosas, lo que debilita la pieza impresa. Esto se puede visualizar en las imágenes de la Figura 2(b), que muestran un detalle de la zona de rotura. Se observan fallas frágiles en todos los casos. Además se pueden distinguir filamentos desgarrados, especialmente los filamentos a $0^{\circ}$ en las probetas $0^{\circ} / 0^{\circ}$ y $0^{\circ} / 90$, lo que evidencia elongación en las zonas sometidas a esfuerzos de tracción durante el ensayo de flexión. A simple vista se pueden reconocer los filamentos individuales que componen el volumen de la probeta.

a)

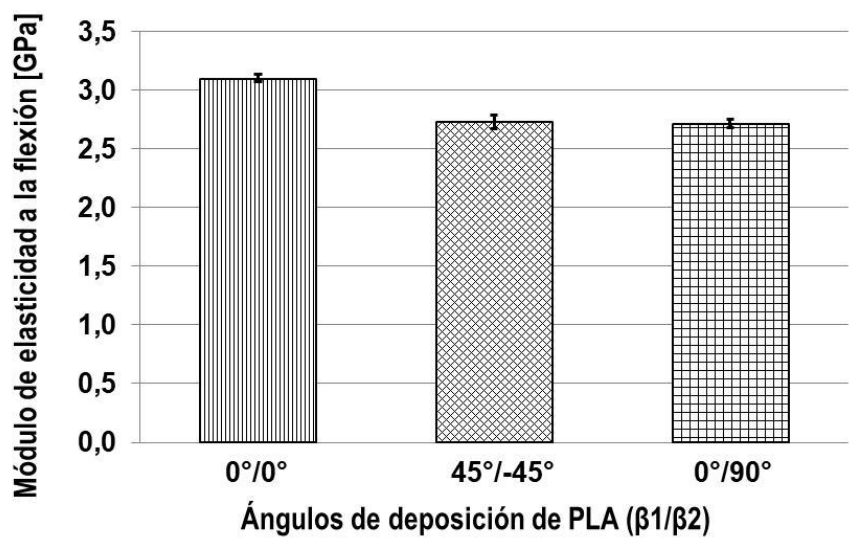

b)
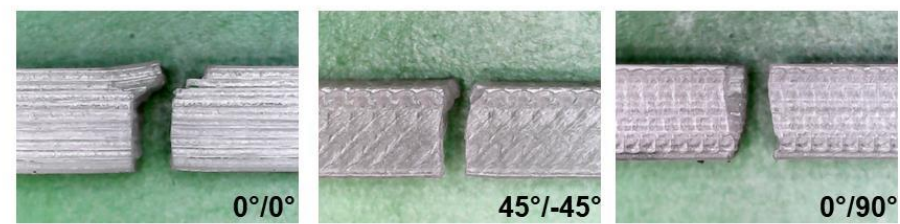

Figura 2: (a) Módulos de elasticidad a la flexión de PLA en función del ángulo de deposición del material. (b) Detalle de la zona de rotura. Imágenes adquiridas con una lupa digital.

En la Figura 3 se compara los módulos de elasticidad a la flexión en probetas de PLA elaboradas con una base de impresión perpendicular al caso anterior, es decir siguiendo el espesor de la probeta (E). Para facilitar la comparación, se incluyen también los resultados ya mostrados en la Figura 2a. Notar que las probetas $0^{\circ} / 90^{\circ} \mathrm{E}$ fueron impresas depositando capas de filamentos de PLA sobre una superficie de menor área que en el caso de las probetas $0^{\circ} / 90^{\circ} \mathrm{A}$. Es decir que la impresión de cada capa individual en $0^{\circ} / 90^{\circ} \mathrm{E}$ concluye en menor tiempo. Por otro lado, la impresión de los filamentos a $90^{\circ}$ se completa en menor tiempo que aquellos a $0^{\circ}$ porque son más cortos. En la gráfica se observan variaciones significativas en los valores medidos. Más aún, un test ANOVA de dos vías revela la existencia de un efecto combinado entre la posición de impresión y el ángulo de deposición. Para el caso de ángulos $0^{\circ} 0^{\circ}$, ambos tipos de probetas - E y A - poseen 
filamentos depositados en la misma dirección de las máximas tensiones. Sin embargo, las probetas $0^{\circ} / 0^{\circ} \mathrm{A}$ presentan un mayor módulo de elasticidad. La diferencia se puede atribuir a la influencia que tiene la adhesión entre capas de PLA con respecto a la posición de la probeta durante el ensayo. En probetas $0^{\circ} / 0^{\circ} \mathrm{E}$, las fuerzas de adhesión entre capas son perpendiculares a las máximas tensiones de flexión, incidiendo en las tensiones de corte, que resultarán menores cuanto menores sean las fuerzas de adhesión. En el caso de probetas $0^{\circ} / 0^{\circ} \mathrm{A}$, durante el ensayo, la probeta se ubica con sus capas de PLA de manera transversal al caso anterior, de modo que las fuerzas de adhesión entre capas contribuyen con mayor eficiencia que en $0^{\circ} / 0^{\circ} \mathrm{E}$, resultando en un mayor módulo de elasticidad a la flexión. Las probetas $45^{\circ} /-45^{\circ} \mathrm{A}$ y $45^{\circ} /-45^{\circ} \mathrm{E}$ presentan valores inferiores a $0^{\circ} / 0^{\circ} \mathrm{A}$, evidenciando que los filamentos están en una geometría más desfavorable con respecto a la dirección de los mayores esfuerzos de tensión.

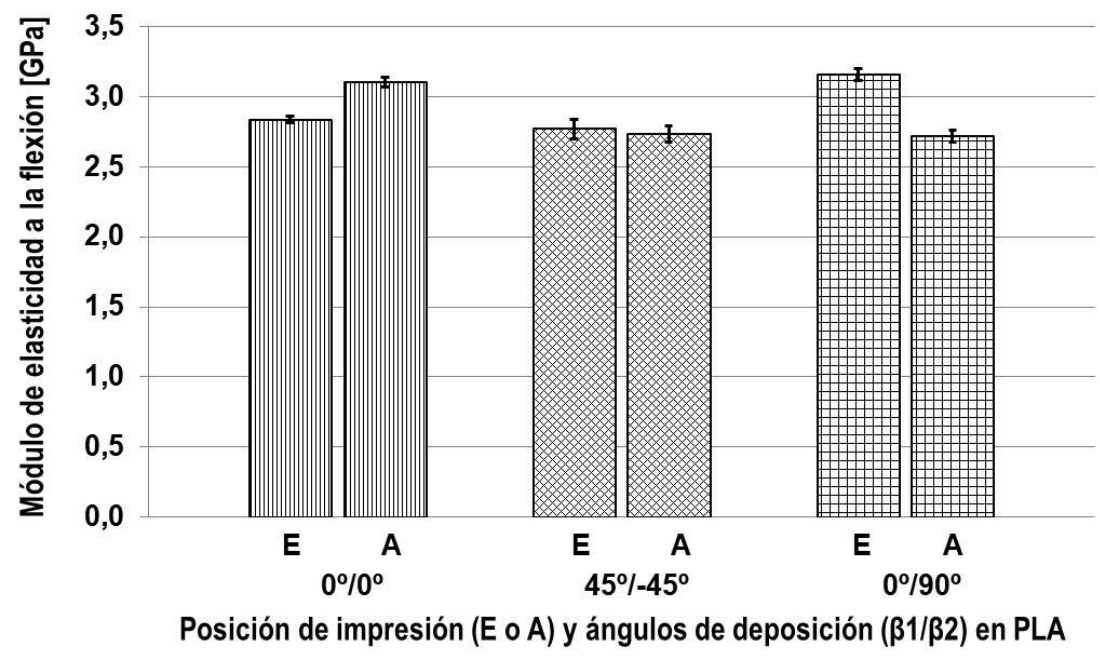

Figura 3: Módulos de elasticidad a la flexión de PLA en función de la posición de impresión. En este caso, se comparan probetas impresas tomando como base la superficie de mayor área (A, ancho) o de menor área (E, espesor), para diferentes ángulos de deposición de filamentos entre capas $(\beta 1 / \beta 2)$.

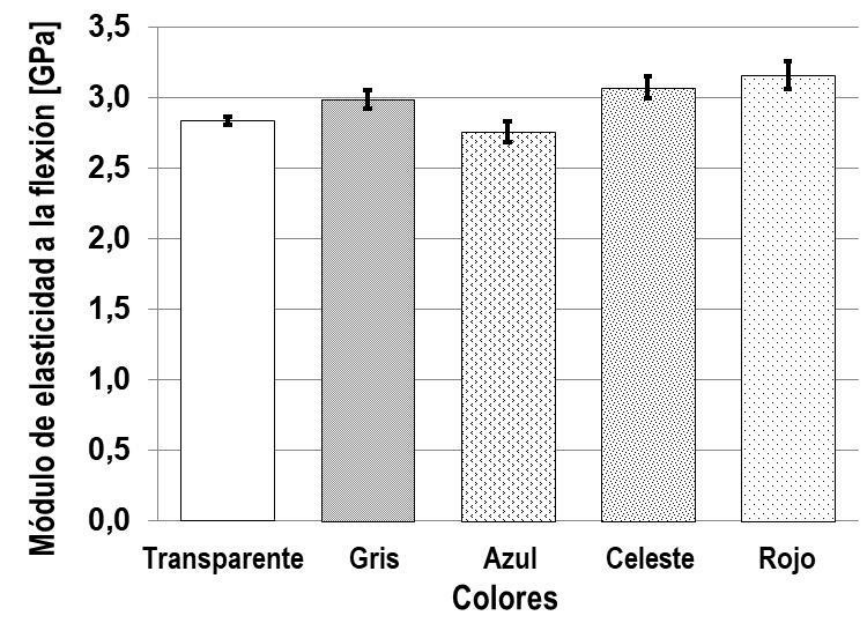

Figura 4: Variación del módulo de elasticidad a la flexión de PLA por efecto de colorantes.

Es conocido que los aditivos pueden influir en el proceso de solidificación de PLA durante el enfriamiento del material fundido [20]-[21] Por ello, es posible que la inclusión de colorantes a la matriz de PLA pueda afectar la forma en que las cadenas poliméricas se adhieren entre sí para formar un sólido y variar las 
características de la pieza final, como por ejemplo calidad de la superficie, viscosidad o cristalinidad [22][23]. Como consecuencia, en este trabajo se analizaron los módulos de elasticidad a la flexión para el caso de probetas construidas con PLA, con diversos aditivos colorantes comerciales. La Figura 4 compara los resultados obtenidos en probetas con y sin aditivos colorantes. Las probetas ensayadas se generaron manteniendo constantes todos los parámetros de impresión: espesor de capa de $0,2 \mathrm{~mm}, 100 \%$ de llenado, velocidad de 32 $\mathrm{mm} / \mathrm{s}$ y la base de impresión es el espesor de la probeta. En la gráfica se puede observar que hay diferencias entre las medias aritméticas, y existe un rango de variación de un 10\% entre los grupos de datos. El análisis estadístico mediante ANOVA de una vía, verifica que existen diferencias significativas entre las medias, con un nivel de confianza del 95\% ( $\mathrm{p}<0,05$ ). Esto confirma la hipótesis de que los aditivos colorantes pueden afectar los módulos de elasticidad de PLA.

La Figura 5 muestra los resultados obtenidos al comparar probetas de PLA rojas utilizando diferentes velocidades de deposición del material. Se muestran resultados para tres velocidades $(24,32$ y $40 \mathrm{~mm} / \mathrm{s})$ y para tres diferentes orientaciones de filamentos. Las probetas presentan mayor módulo de elasticidad a la flexión cuando los filamentos se encuentran en la dirección $0^{\circ} / 0^{\circ}$ y se depositan lentamente. Se observa que a $0^{\circ} / 0^{\circ}$ y $0^{\circ} / 90^{\circ}$, los valores disminuyen a medida que la velocidad aumenta, siendo este efecto más evidente para filamentos a $0^{\circ} / 0^{\circ}$ que a $0^{\circ} / 90^{\circ}$. De manera alternativa, para el caso de filamentos que se depositan a $45^{\circ} / 45^{\circ}$, la velocidad de impresión no tiene una influencia significativa. Para máximas velocidades de deposición, el módulo de elasticidad a la flexión tiende al valor mínimo registrado a lo largo de este trabajo, que es equivalente para los tres ángulos de deposición. Un fenómeno semejante se ha observado en los casos con otros pigmentos. Probablemente, el hecho de que el módulo aumente al disminuir la velocidad se deba a que los filamentos poliméricos están más tiempo parcialmente fundidos y pueden adherirse mejor durante el proceso de impresión. Este hecho es más notorio cuando hay capas de material con filamentos a $0^{\circ} / 0^{\circ}$ porque existe mayor área de contacto entre dichos filamentos. Los datos muestran que tanto la velocidad como el ángulo de deposición son significativos para el caso de PLA. Además, al evaluar mediante ANOVA de dos vías si existe un efecto combinado entre estos dos factores, se concluye que no hay interacción entre ellos ( $p$ $>0,05)$.

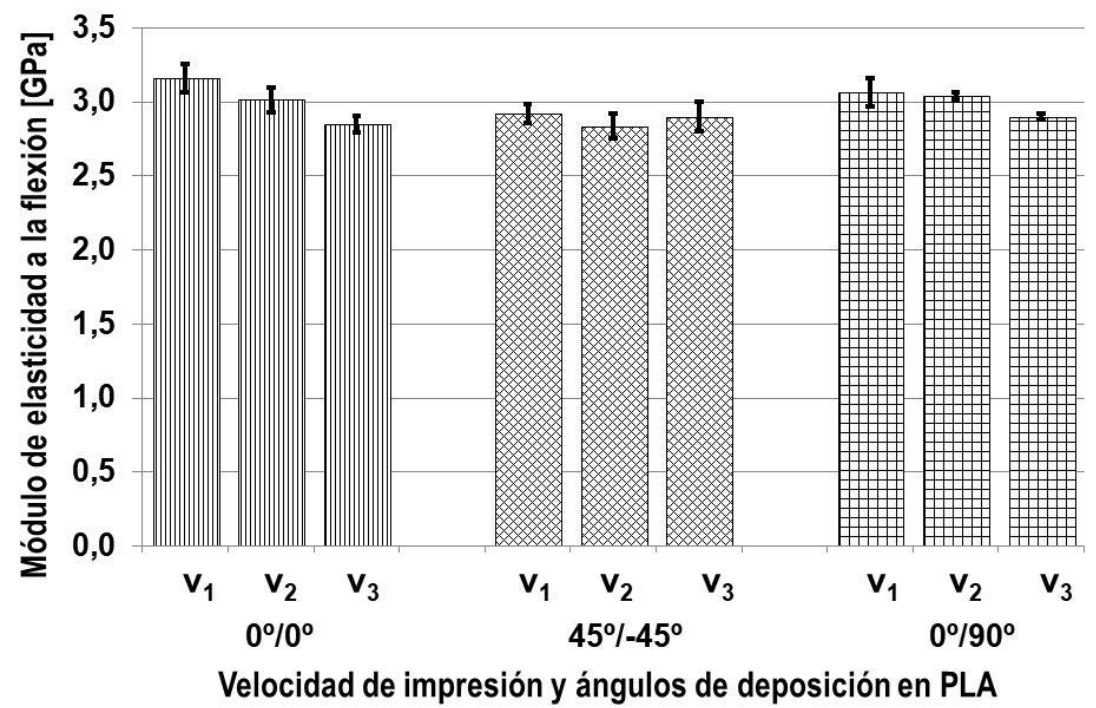

Figura 5: Módulos de elasticidad a la flexión de PLA en función del ángulo de deposición del material y de la velocidad de impresión (v), donde $\mathrm{v}_{1}<\mathrm{v}_{2}<\mathrm{v}_{3}$.

La Figura 6 muestra los resultados del módulo de elasticidad a la flexión obtenidos con probetas de HIPS. En la misma se cotejan (i) los tres regímenes de velocidad de impresión, (ii) los tres colores elegidos y (iii) las tres disposiciones angulares para la clase de probeta de base A. En términos generales, se observan algunas similitudes al caso del PLA. A medida que aumenta la velocidad de impresión, disminuye el módulo de elasticidad. Los valores del módulo de elasticidad son mayores cuando la disposición de impresión es 0/0, es decir, cuando los filamentos son impresos en forma paralela a las direcciones de las máximas tensiones, tomando como base de impresión la superficie de mayor área. Además, los aditivos colorantes inducen varia- 
ciones estadísticamente significativas.

En la Figura 7 se compara la influencia de la posición de impresión en el módulo de elasticidad, es decir, el efecto de usar probetas de tipo A o E. Nótese que en la mayoría de los casos, la impresión efectuada con el tipo A, da los mayores valores en el módulo de elasticidad para un color y disposición angular específica. Si bien la Figura 7 resume los valores registrados a la velocidad de impresión más lenta $\left(\mathrm{v}_{1}=24 \mathrm{~mm} / \mathrm{s}\right)$, en las otras dos velocidades estudiadas se observa un comportamiento similar. El análisis estadístico de los resultados indica que el efecto de la disposición longitudinal de los filamentos impresos resulta en un mayor módulo de elasticidad frente a las otras dos disposiciones evaluadas. La velocidad de impresión es importante y significativa, en tanto que para obtener mejores respuestas la velocidad de impresión debe ser la más baja. La dirección de impresión también es un factor importante, siendo el tipo A el que resulta generalmente en módulos de elasticidad más altos.

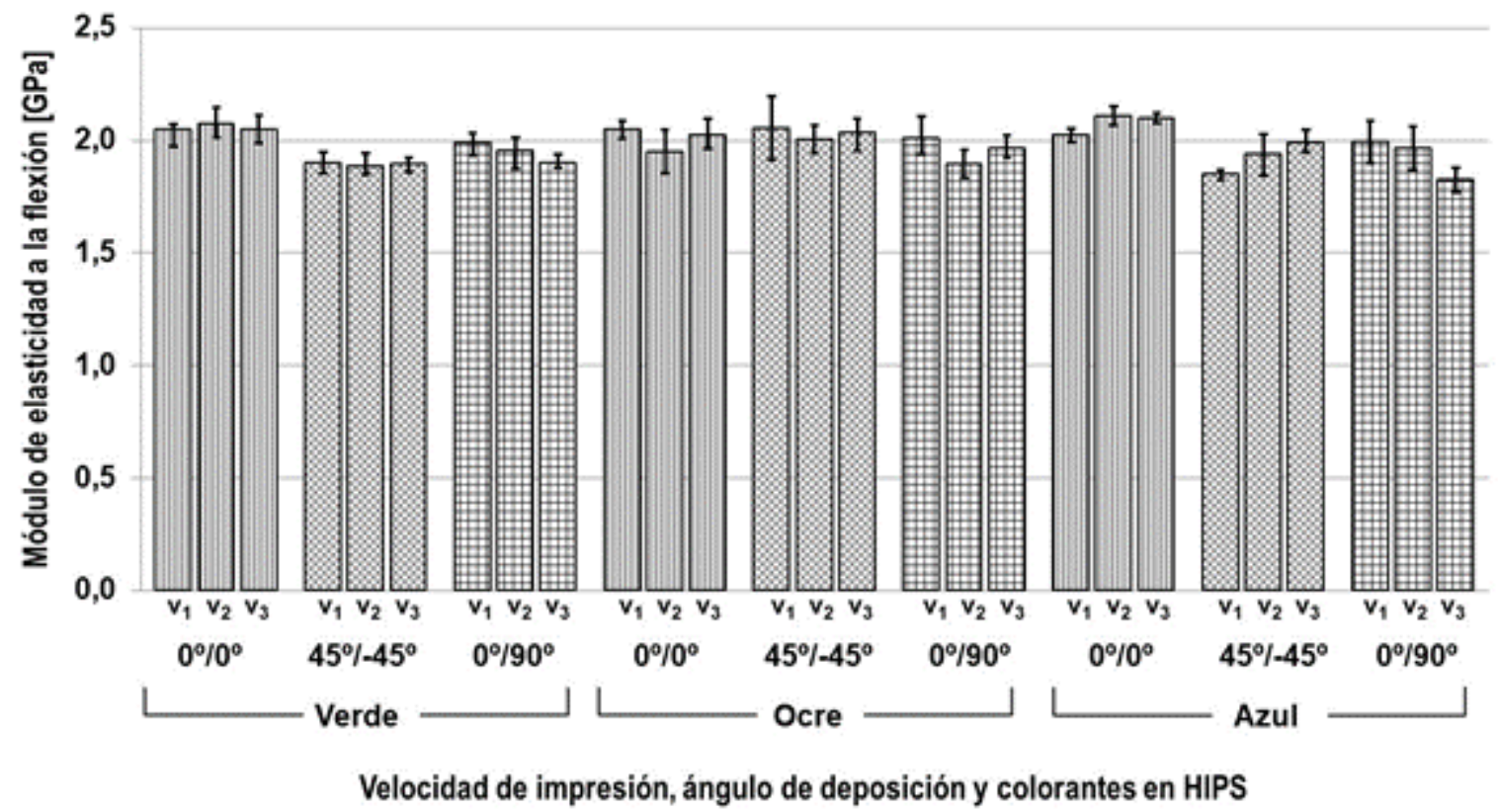

Figura 6: Módulos de elasticidad a la flexión de HIPS en función de la velocidad de impresión ( $\mathrm{v}$, donde $\mathrm{v}_{1}<\mathrm{v}_{2}<\mathrm{v}_{3}$ ), ángulo de deposición del material y coloración empleadas.

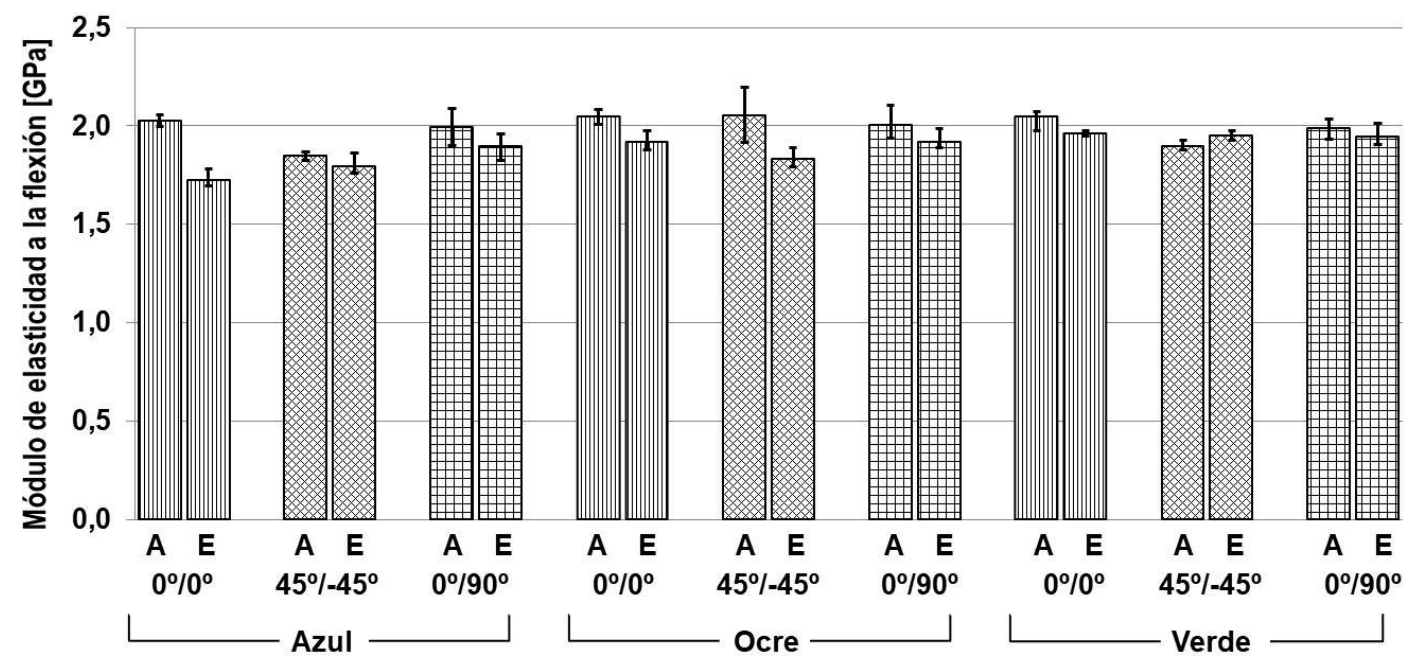

Posición de impresión (E o A), ángulos de deposición ( $\beta 1 / \beta 2)$ y colorantes en HIPS

Figura 7: Módulos de elasticidad a la flexión de HIPS en función de la base de impresión (superficie de mayor área, A, o de menor área, E) y de distintos ángulos de deposición, para tres colorantes. Caso de $\mathrm{v}_{1}=24 \mathrm{~mm} / \mathrm{s}$. 
En la Figura 8 se muestran los resultados obtenidos con probetas de Nylon, variando el ángulo de deposición entre capas, la velocidad de impresión y la presencia de colorantes. Los valores están en concordancia con las tendencias registradas en probetas de PLA y de HIPS. Nuevamente, el máximo módulo de elasticidad corresponde al caso de filamentos impresos a $0^{\circ} / 0^{\circ}$, disminuyendo para $45^{\circ} / 45^{\circ}$ y para $0^{\circ} / 90^{\circ}$. A su vez, la velocidad de impresión para este material juega un papel crucial. Cabe notar que en la Figura 8 no se incluyen los casos correspondientes a la velocidad de impresión $\mathrm{v}_{3}=40 \mathrm{~mm} / \mathrm{s}$. Esto se debe a que se observó delaminación en varias probetas de esta serie, y una dispersión no desdeñable en las pocas probetas que sí pudieron ser ensayadas. Todo esto hizo que sea necesario descartar las probetas de Nylon impresas a mayor velocidad. Una situación similar ocurrió al intentar imprimir probetas tomando como base la posición E. Al ser la base de impresión muy pequeña, muchas probetas resultaron defectuosas, por lo que los datos de módulo de elasticidad que resulten de ellas no son representativos del material.

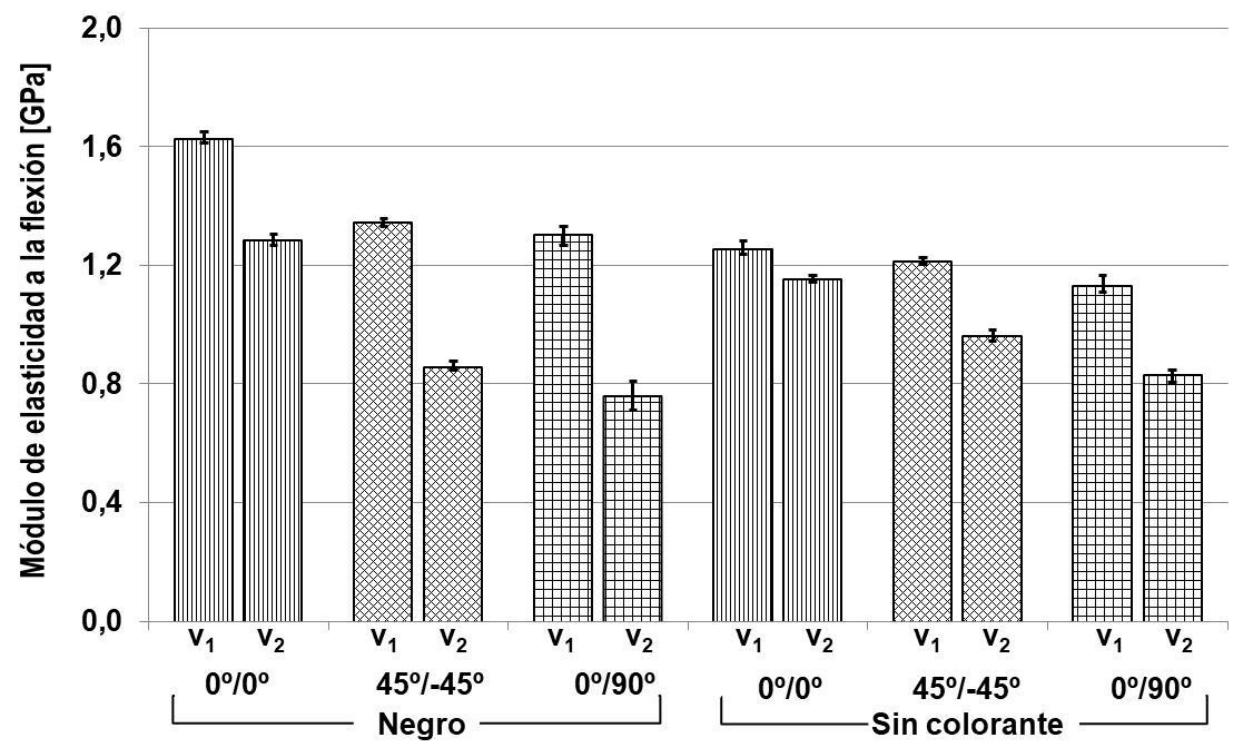

Velocidad de impresión y ángulos de deposición ( $\beta 1 / \beta 2)$ en Nylon, con y sin colorante

Figura 8: Módulos de elasticidad a la flexión de Nylon en función del ángulo de deposición del material y la velocidad de impresión ( $\mathrm{v}$, donde $\mathrm{v}_{1}<\mathrm{v}_{2}$ ), para probetas con colorante (negro) y sin colorante.

Si se comparan los máximos valores aquí obtenidos para cada material, con sus homólogos sin procesar por MDF [24]-[26] y con los disponibles en otros estudios similares [27]-[28], se arriba a los datos resumidos en la Tabla 5. En el caso de PLA, el proceso de impresión 3D induce una disminución significativa del módulo elasticidad a la flexión. En el caso de Nylon, el máximo valor alcanzado por las probetas impresas se mantiene dentro del rango reportado para sus homólogos no impresos, pero está próximo al límite inferior. En el caso de HIPS, se observa que la impresión 3D no implica una disminución con respecto a los valores conocidos para este polímero. Además, de los tres materiales ensayados, HIPS es el que presentó menor variabilidad con respecto a las variables de impresión analizadas.

Tabla 5: Comparación del Módulo de elasticidad a la flexión en piezas MDF (presente estudio) y la literatura.

\begin{tabular}{l|l|l|l}
\hline \multirow{2}{*}{ MATERIAL } & \multicolumn{3}{|l}{ MÓDULO DE ELASTICIDAD A LA FLEXIÓN [GPa] } \\
\cline { 2 - 4 } & Presente MDF & Otros, no MDF [22]-[24] & Otros MDF [25]-[26] \\
\hline PLA & $2,897-3,169$ & 4,000 & $3,395-3,516$ \\
\hline HIPS & $1,985-2,105$ & $1,900-2,300$ & ---- \\
\hline Nylon & $1,287-1,630$ & $1,200-3,100$ & ---- \\
\hline
\end{tabular}

Materiales como HIPS y Nylon son susceptibles a la absorción de humedad, lo cual provoca alteraciones importantes en la calidad de impresión y la calidad de las propiedades elásticas resultantes. Esto se evi- 
denció principalmente en el caso de Nylon. Si bien, los materiales utilizados fueron sometidos a procesos de disecado y eliminación de humedad (métodos que son considerados convencionales en la técnica MDF) se ha observado una diferencia notoria entre los valores del módulo de elasticidad para el color negro y sin pigmentación. También se presentaron dificultades en la impresión de las probetas a mayores velocidades, con casos muy frecuentes de delaminación y/o terminación geométrica no aceptable.

\section{CONCLUSIONES}

En este trabajo se presentó un estudio sobre las propiedades mecánicas determinadas en piezas poliméricas, generadas por impresión 3D. En particular, se comparó el módulo de elasticidad a la flexión en materiales de uso comercial - PLA, Nylon y HIPS - mediante ensayos de probetas conformadas por el método de modelación por deposición de filamento. Se evaluó el efecto que tiene la variación de diversos parámetros constructivos durante el proceso de impresión, tales como: orientaciones del filamento en la impresión (ángulo de deposición), velocidad de impresión, presencia de aditivos colorantes y posición de la probeta con respecto a la ubicación de la boquilla de impresión (base de impresión).

En particular, al variar el ángulo de orientación de filamentos, se verificó que los máximos módulos se obtienen cuando las probetas se imprimen con todos los filamentos paralelos a la dirección de las máximas tensiones. Este comportamiento se observó en todos los materiales analizados.

Además, otro de los parámetros que evidenció variaciones significativas, es la velocidad de impresión. Se comprobó que el módulo de elasticidad disminuye al aumentar la velocidad de impresión. No obstante, se observó que en el caso de PLA, las variaciones son despreciables cuando los filamentos se encuentran a $45 \%$ $45^{\circ}$.

La base de impresión que se tome como geometría inicial a partir de la cual generar la probeta, es un parámetro que puede influir en la resistencia a esfuerzos de flexión. En general, se encontró una mejor performance cuando las probetas se imprimieron adoptando la base de impresión de mayor área.

Se registró que los aditivos colorantes también pueden afectar significativamente el desempeño mecánico de los tres materiales estudiados.

Se concluye que el módulo de elasticidad a la flexión puede variar significativamente en función de todos los parámetros de impresión analizados. Consecuentemente, se puede afirmar que el método de modelación por deposición de filamento da como resultado piezas anisótropas.

\section{AGRADECIMIENTOS}

Este trabajo se realizó con el financiamiento de la Universidad Tecnológica Nacional a través del proyecto PID 4285 TUN, y el apoyo de CONICET (Argentina).

\section{BIBLIOGRAFÍA}

[1] CANESA E., FONDA C., GENARO M., Low cost 3D-printing, Ed. Intl. Center Theor. Physics, 2013.

[2] WIMPENNY, D., PANDEY, P., JYOTHISH KUMAR, L. Advances in 3D Printing \& Additive Manufacturing Technologies, Ed. Springer Science Business Media Singapore, 2017.

[3] AHN S., MONTERO, M., ODELL, D., et al., "Anisotropic material properties of fused deposition modeling ABS”, Rapid Prototyping Journal, v. 8 n. 4, pp. 248-257, 2002.

[4] LI L., SUN Q., BELLEHUMEUR C., GU P. "Composite modelling and analysis for fabrication of FDM prototypes with locally controlled properties", Journal of Manufacturing Processes, v. 4 n. 2, pp.129-141, 2001.

[5] RODRIGUEZ, J., THOMAS, J., RENAUD, J. "Mechanical behavior of acrylonitrile butadiene styrene (ABS) fused depositions materials. Experimental investigation”, Rapid Prototyping Journal, v. 7 n. 3, pp. 148-158, 2001.

[6] SYAMSUZZAMAN, M., MARDI, N., FADZIL, M., et al., "Investigation of layer thickness effect on the performance of low-cost and commercial fused deposition modelling printers", Materials Research Innovations. v. 18 n. 6, 485-489, 2014.

[7] VAEZI, M., CHUA, C. "Effects of layer thickness and binder saturation level parameters on 3D printing process", International Journal of Advanced Manufacturing Technology, v. 53, pp. 275-284, 2011. 
[8] ZELENY, P., SAFKA, J., ELKINA, I. "The mechanical characteristics of 3D printed parts according to the build orientation", Applied Mechanics and Materials, v. 474, pp. 381-386, 2014.

[9] TYMRAK B.M., KREIGER M., PEARCE J.M., "Mechanical properties of components fabricated with open-source 3-D printers under realistic environmental conditions", Materials and Design, v. 58, pp. 242-246, 2014.

[10] LANZOTTI, A., GRASSO, M., STAIANO, G., et al., "The impact of process parameters on mechanical properties of parts fabricated in PLA with an open-source 3-D printer", Rapid Prototyping Journal, v. 21 n. 5 , 2015.

[11] DELGADO CAMACHO, D., et al., "Applications of additive manufacturing in the construction industry - A forward-looking review", Automation in Construction, v 89, pp. 110-119, 2018.

[12] WANG, X., JIANG, M., ZHOU, Z., et al., "3D printing of polymer matrix composites: A review and prospective”, Composites Part B: Engineering, v. 110, pp. 442-458 , 2017.

[13] NGO, T.D., KASHANI, I. G., NGUYEN, K.T.Q., et al., “Additive manufacturing (3D printing): A review of materials, methods, applications and challenges", Composites Part B: Engineering, v. 143, pp. 172196, 2018

[14] TORRADO, A.R., ROBERSON, D.A. "Failure Analysis and Anisotropy Evaluation of 3D-Printed Tensile Test specimens of different Geometries and Print Raster Patterns", Journal Failure Analysis and Prevention, v. 16, pp. 154-164, 2016.

[15] CHACON, J.M, CAMINERO, M.A., GARCIA-PLAZA, E., et al., "Additive manufacturing of PLA structures using fused depositing modelling: effect of process parameters on mechanical properties and their optimal selection", Materials and Design, v. 124, n. 15, pp. 143-157, 2017.

[16] SOOD, A.K., OHDAR, R.K., MAHAPATRA, S.S., "Parametric appraisal of mechanical property of fused deposition modelling processed parts”, Materials and Design, v. 31, pp. 285-295, 2010.

[17] FORSTER A.M., "Materials testing standars for additive manufacturing of polymer materials: State of the art and standards applicability”, In: Report NISTIR 8059, National Institute of Standards and Technology, U.S. Departament of Commerce. http://dx.doi.org/10.6028/NIST.IR.8059 , 2015.

[18] REPLIKAT http://replikat.com.ar/productos/impresora-3d-argentina-replikat-m5 , accedido marzo 2018.

[19] ISO 178, ISO. "Plastics: Determination of flexural properties", 2003.

[20] TSUJI, H., TAKAI, H., FUKUDA, N., et al., "Non - Isothermal Crystallization Behavior of Poly(Lactic acid) in the Presence of Various Additives", Macromolecular Materials and Engineering, v. 291, n.4, pp. 325-335, 2006.

[21] BILAL, M.B., et al., "A study of the structural changes during the dyeing process of IngeoTM fibers of poly(lactic acid)", Textil Research Journal, v. 81, n. 8, pp. 838-846, 2011.

[22] VALERGA, A. et al., "Influence of PLA Filament Conditions on Characteristics of FDM Parts", Materials, v. 11, n. 8, pp. 1322, 2018.

[23] WITTBRODT, B., PEARCE, J.M. "The effects of PLA color on material properties of 3-D printed components", Additive Manufacturing, v. 8, p.p. 110-116, 2015.

[24] MARKEITFROM. https://www.makeitfrom.com/material-properties/Polylactic-Acid-PLA-Polylactide , accedido mayo de 2019.

[25] MARKEITFROM. https://www.makeitfrom.com/material-group/Polyamide-Nylon-Plastic , accedido mayo de 2019

[26] CROW@ Polymer Science. https://polymerdatabase.com/Commercial\%20Polymers/PS2.html , accedido mayo de 2019.

[27] OKSMANA, K., SKRIFVARS, M., SELIN, J.-F. "Natural fibres as reinforcement in polylactic acid (PLA) composites”, Composites Science and Technology, v. 63, pp. 1317-1324, 2003.

[28] BIJARIMI, M., AHMAD, S., RASID, R. "Mechanical, Thermal and Morphological Properties of PLA/PP Melt Blends", International Conference on Agriculture, Chemical and Environmental Sciences (ICACES'2012) Dubai, Emiratos Arabes Unidos, Octubre 2012. 


\section{ORCID}

Darío Stechina

https://orcid.org/0000-0002-1619-6509

Sandra Marina Mendoza

http://orcid.org/0000-0003-1691-0052

Héctor Daniel Martín

http://orcid.org/0000-0002-8525-2471

Claudio Norberto Maggi

http://orcid.org/0000-0003-0033-6287

Marcelo Tulio Piovan

http://orcid.org/0000-0001-9464-1076 